\title{
DISTRIBUTIONS OF BORON AND PHOSPHORUS IMPLANTED IN SILICON IN THE ENERGY RANGE 0.1-1.5 MeV
}

\author{
S. OOSTERIIOFF * \\ Twente University of Technology, Department of Electrical Engineering, P.O. Box 217, 7500 AE Enschede, The Netherlands
}

Received 24 June 1987 and in revised form 8 October 1987

Boron and phosphorus were implanted in p-type and n-type silicon wafers in the energy range from 0.1 to $1.5 \mathrm{MeV}$. Three different methods were used to determine the distribution of the ions: SIMS, CV and NRA. The results were fitted to a Pearson IV distribution in order to extract moments for describing the distributions analytically. The projected ranges agree well with the theoretical values. Deviations are observed at higher energies. Projected range standard deviations are significantly greater than the tabulated values. The skewness clearly deviates from available tabulated data, although the same trend is observed.

\section{Introduction}

Interest in the application of high-energy ion implantations in semiconductor technology is increasing. The depth range of the implanted ions can be enlarged to several microns. An important application is found in CMOS technology. So-called retrograde wells can be made which have smaller lateral and vertical dimensions than wells made with standard implantation-diffusion techniques. Also latch-up prevention is easier with retrograde wells [1-4].

For the effective use of high energy ion implantation accurate data are required concerning the range distributions of these implantations. It is the purpose of our research to provide experimental verification of the tabulated moments of the implanted ion distributions. Three different measuring methods have been used: capacitance-voltage (CV) profiling, secondary ion mass spectrometry (SIMS) and nuclear reaction analysis (NRA). The experimental results are fitted to a Pearson IV distribution $[5,6]$ from which four parameters, the moments of the distribution, are then extracted. Once these moments have been obtained, it is easy to implement them in programs such as SUPREM [7] or ICECREAM [8], in order to obtain reliable process simulations.

\section{Experimental procedure}

\subsection{Sample preparation}

A $500 \mathrm{kV}$ HVEE ion accelerator was applied for the implantations. Using doubly- and triply-charged ions

\footnotetext{
* Present address: Philips Research Laboratories, P.O. Box $80.000,5600$ JA Eindhoven, The Netherlands.
}

experimental results could be obtained in the energy range of $0.1-1.0 \mathrm{MeV}$ for boron and of $0.1-1.5 \mathrm{MeV}$ for phosphorus. The installation of an extra mass separator, directly after the accelerating tube, appeared absolutely necessary in order to get reproducible and reliable results because a relatively large number of the multiplecharged ions capture one or two electrons in the accelerator tube. Monocrystalline silicon wafers (100) were implanted with ion doses of $10^{12} / \mathrm{cm}^{2}$ for CV measurements and $2 \times 10^{14}$ and $10^{15} / \mathrm{cm}^{2}$ for SIMS and NRA measurements.

Due to the low yield of triply charged phosphorus ions, only $1.5 \mathrm{MeV}$ samples were prepared for the SIMS measurements using a dose of approximately $1.3 \times 10^{14}$ ions $/ \mathrm{cm}^{2}$. In the energy range $0.1-0.5 \mathrm{MeV}$, also samples were prepared with a dose of $10^{16} / \mathrm{cm}^{2}$.

During implantation the samples were tilted $7^{\circ}$ and rotated approximately $27^{\circ}$ in order to minimize channeling. This is close to the $7^{\circ}$ tilt and $30^{\circ}$ twist that is recommended by Turner et al. [9].

\subsection{Differential capacitance measurements}

Every sample was extra implanted with the appropriate dopant to a dose of $5 \times 10^{11} / \mathrm{cm}^{2}$ at $20 \mathrm{keV}$ in order to decrease the depletion depth at zero bias and therefore extending the measured depth range towards the surface.

Three quarters of every wafer were subsequently implanted with a dose of $10^{12} / \mathrm{cm}^{2}$ at one of the chosen energies. The remainder of every wafer was used as a standard to obtain the implanted profile by subtracting the bulk doping and the $20 \mathrm{keV}$ implantation from the measured profile. This procedure is illustrated in fig. 1, where a $100 \mathrm{keV}$ boron implantation is used.

After the implantation the samples were boiled in $65 \%$ nitric acid for 10 minutes and rinsed in de-ionized 
$1 E+17-$

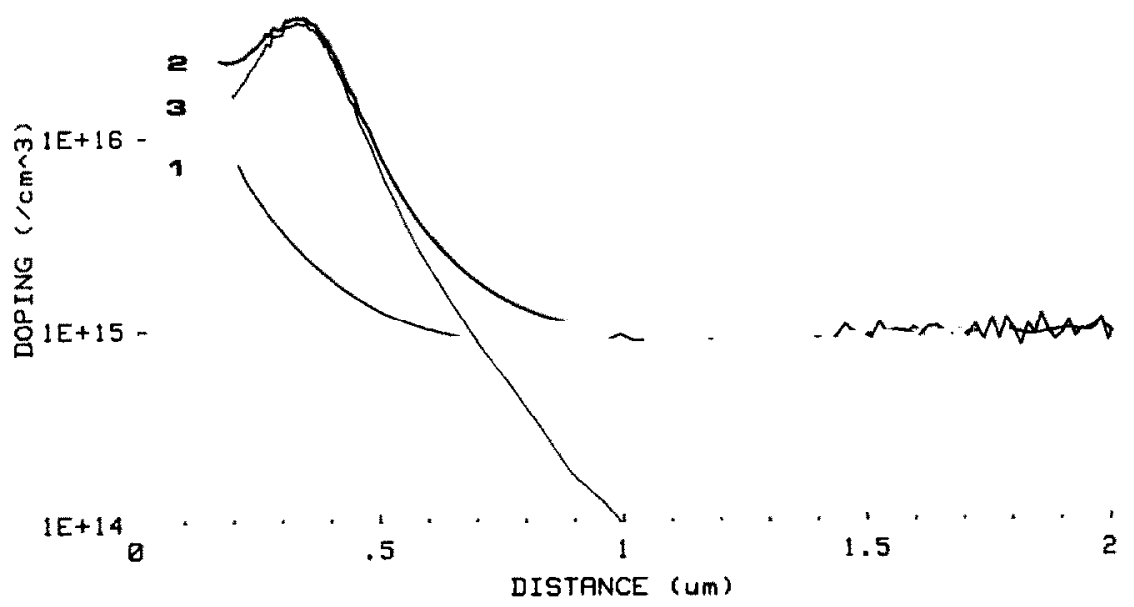

Fig. 1. Illustration of the determination of the $\mathrm{CV}$ profile. 1: $20 \mathrm{keV}$ implantation + background concentration; $2: 20 \mathrm{keV}+100 \mathrm{keV}$ implantation with background concentration; 3 : the resulting $100 \mathrm{keV}$ doping profile.

water for the same period. In order to activate the implanted impurities, the samples were annealed at $800^{\circ} \mathrm{C}$ in a dry $\mathrm{N}_{2}$ ambient for $15 \mathrm{~min}$. This suffices for complete activation as was shown before [10].

$\mathrm{CV}$ measurements were performed with an HP-testsystem in combination with an MSI $\mathrm{Hg}-2 \mathrm{C}-5$ double column mercury probe. The impurity profiles were calculated from the average of $5 \mathrm{CV}$ measurements, in order to minimize the influence of statistical fluctuations.

\subsection{SIMS measurements}

The SIMS measurements were performed for us at the Surface Science Laboratory of the University of Western Ontario, Canada, using a CAMECA IMS-3f ion microscope. Boron was measured, alternately with ${ }^{30} \mathrm{Si}$ in order to monitor the sputter rate, using a primary beam of positive oxygen molecules. In the case of phosphorus high mass-resolution was used $(M / \Delta M \approx$ 3900) using a primary beam of $\mathrm{Cs}^{+}$-ions. Depth scales were obtained by measuring the total crater-depths with a Talystep step height meter.

\subsection{NRA measurements}

Boron and phosphorus were also profiled using a nuclear reaction technique. These measurements were performed at the Materials Science Centre of the State University of Groningen using a $6 \mathrm{MeV}$ Van der Graaff accelerator.

For profiling boron, the ${ }^{11} B(\alpha, n){ }^{14} N$ reaction was used with $E_{\alpha}=2060 \mathrm{keV}$, while for phosphorus the ${ }^{31}(\alpha, \mathrm{p}){ }^{34} \mathrm{~S}$ reaction was used with $E_{\alpha}=3047 \mathrm{keV}$.

The detection limit of this method is high (approximately $10^{19} / \mathrm{cm}^{3}$ ) and therefore the resolution of the profiles obtained with this method was rather poor. These profiles were not used in the fitting procedure for the extraction of moments, but only for the determination of the position of the peak of the impurity distribution. In this way the method was used to check the depths obtained by the other two methods. A detailed description of this method has been published [11].

\subsection{The fit procedure}

Once the profiles had been obtained, they were fitted to a Pearson IV distribution, because this type of distribution allows a good fit to experimental profiles $[5,6]$ and the resulting moments can easily be implemented in process-simulation programs $[7,8]$.

The fitting was done using the specially made computer program IMPFIT. This program uses a nonlinear least squares data fitting procedure as described by Fletcher [12].

\section{Results and discussion}

\subsection{Boron implantations}

Some of the implantation profiles for boron are shown in figs. 2 and 3 as determined with SIMS and CV measurements respectively. From these graphs it can be 


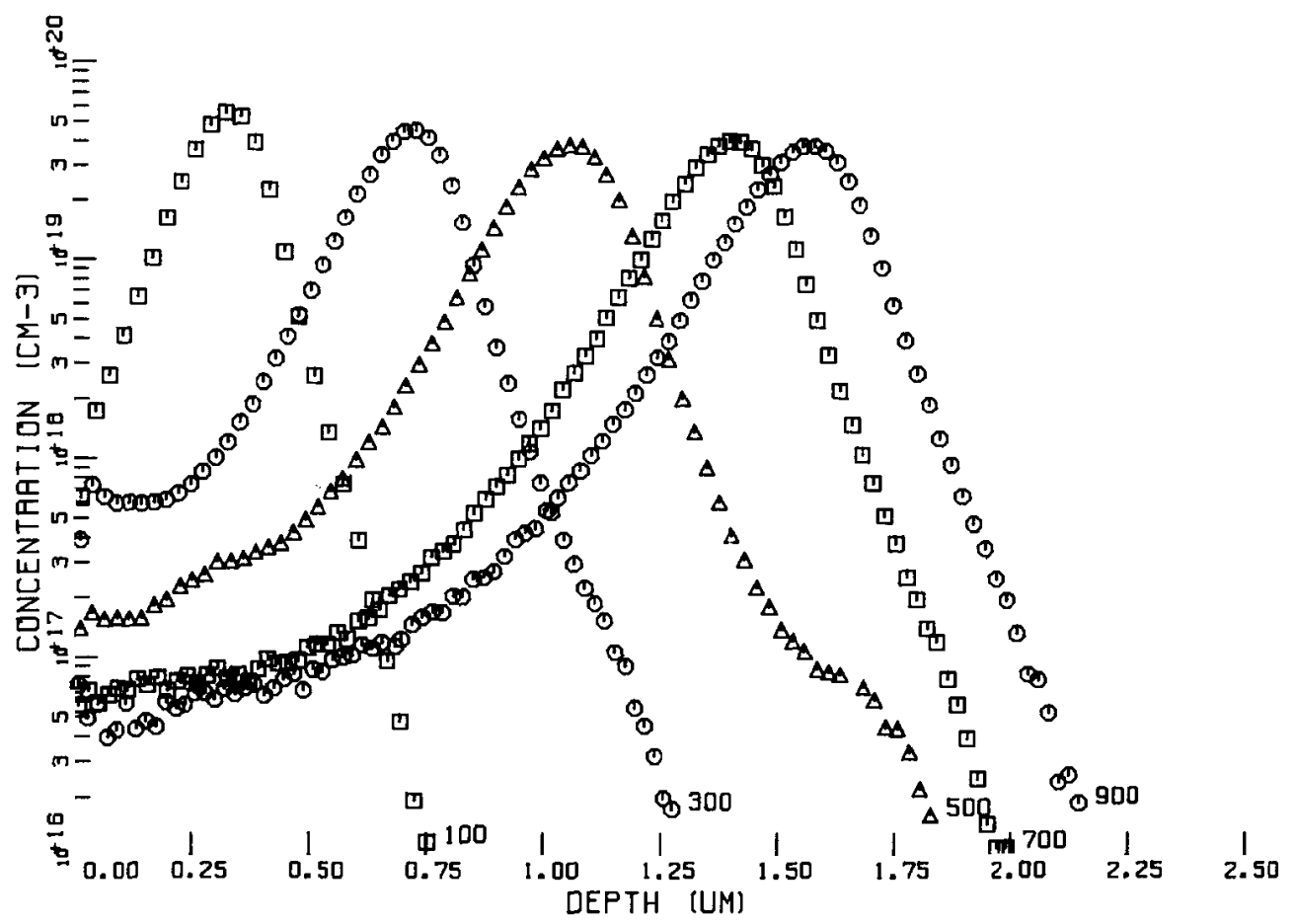

Fig. 2. Impurity distributions of $10^{15} / \mathrm{cm}^{2} 100,300,500,700$ and $900 \mathrm{keV}$ boron implantations in monocrystalline silicon as determined by SIMS.

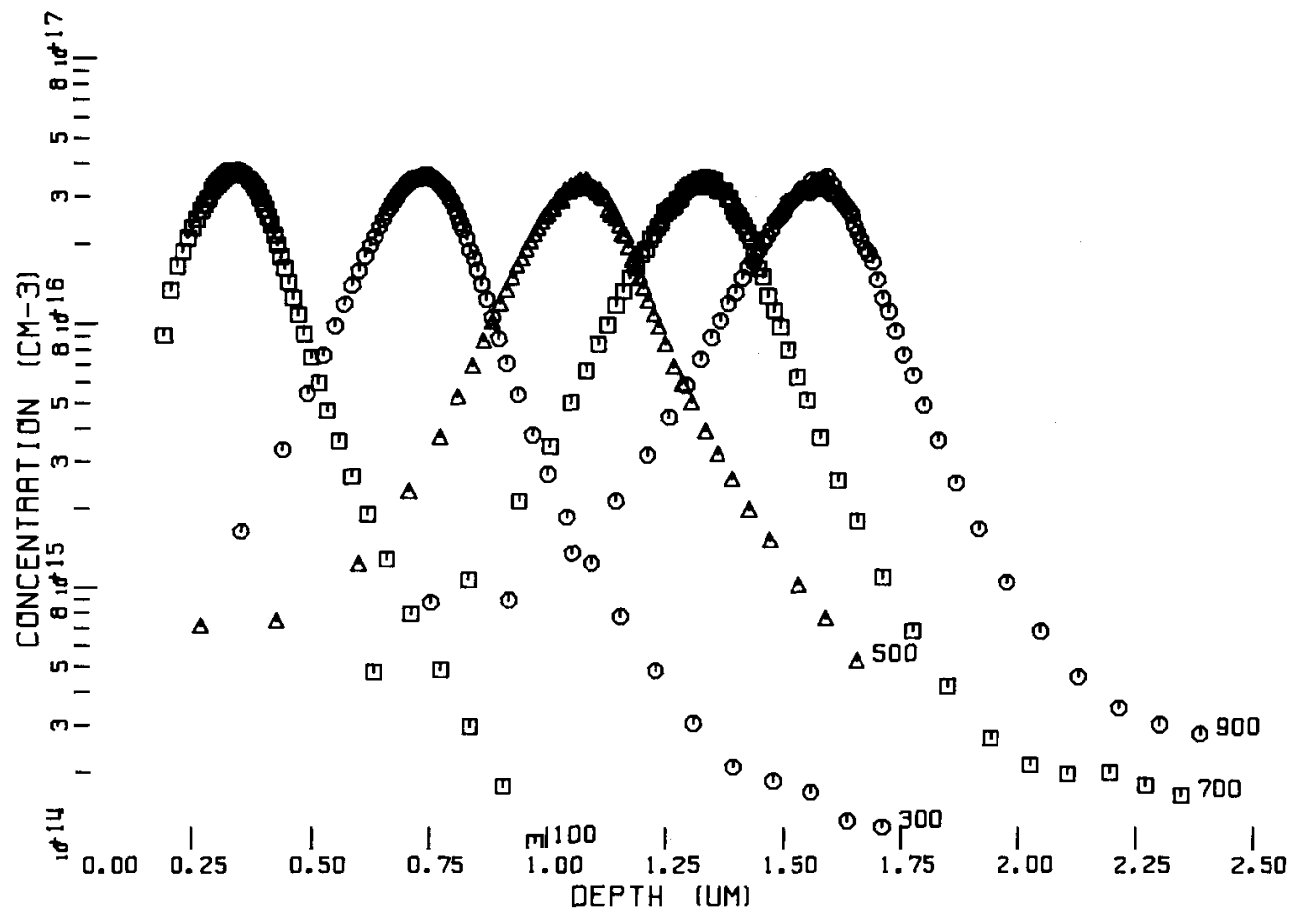

Fig. 3. Impurity distribution of $10^{12} / \mathrm{cm}^{2} 100,300,500,700$ and $900 \mathrm{keV}$ boron implantations in monocrystalline silicon as determined from CV measurements. 


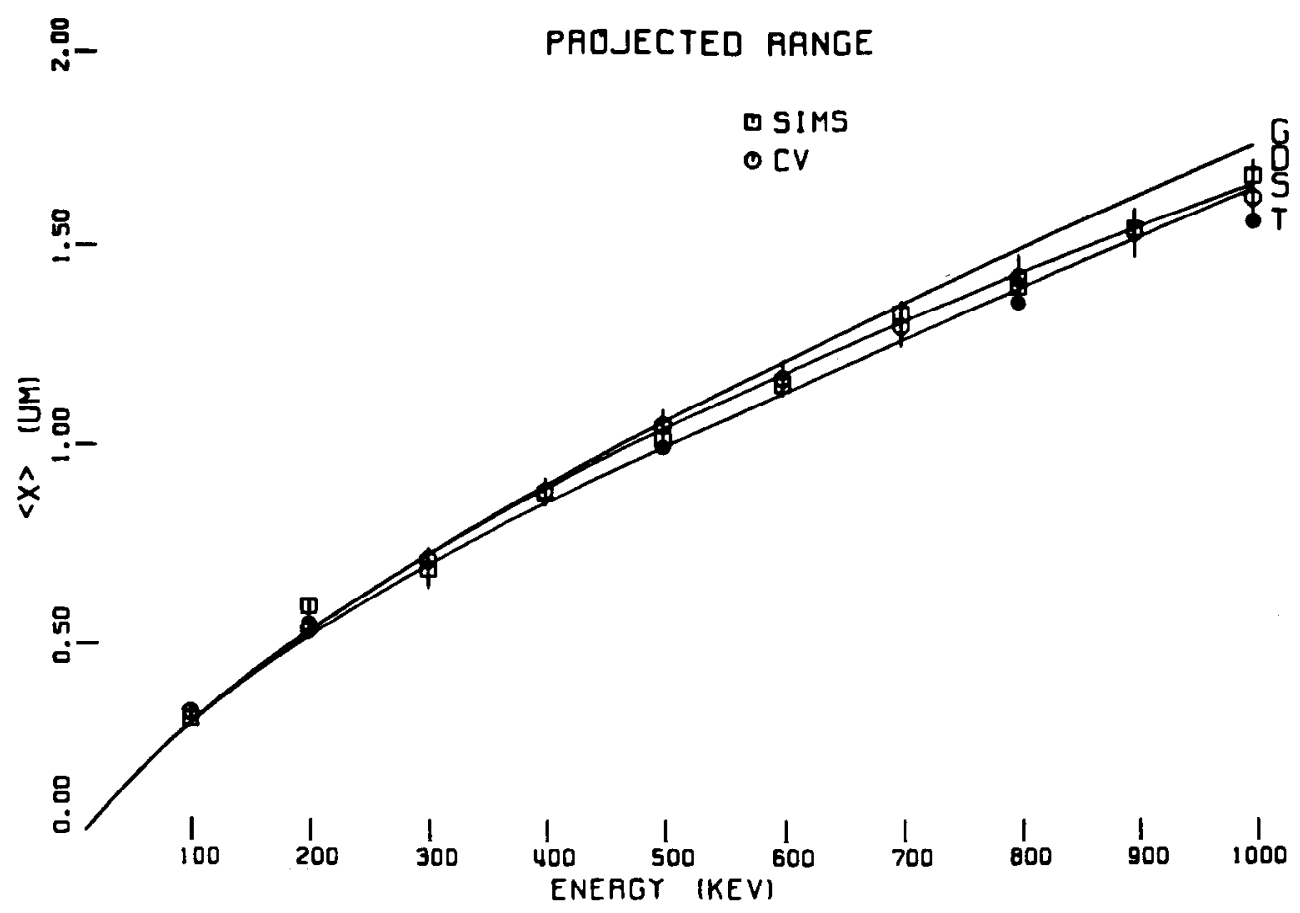

Fig. 4. Projected range of boron in silicon as a function of implantation energy, compared with tabulated data S: Smith [16], G: Gibbons et al. [17], D: DIMUS fit $k / k_{\mathrm{L}}=1.57, p=0.49$. T: TRIM 85 calculations.

concluded that differences arise between corresponding implantations. Apart from the difference in concentration resulting from different doses used: $10^{12} / \mathrm{cm}^{2}$ for the CV measurements and $10^{15} / \mathrm{cm}^{2}$ for the SIMS measurements, there are other marked differences.

The $\mathrm{CV}$ profiles show penetrating tails, unlike the SIMS profiles. Seidel [13] found similar tails in CV profiles and concluded that these tails are actually present in the impurity distribution since they do not change significantly when measured at low temperatures $(90 \mathbf{K})$. Wu et al. [14] state that in the case of implanted profiles the Debye length should be taken into account and that "at least a fraction of the tail observed by the $\mathrm{CV}$ technique is not due to the actual presence of a tail in the distribution". We therefore carried out the fitting procedure with and without taking into account the data points of the tail. Points which obviously resulted from channeling were excluded from the fitting procedure. It turned out that the data of the tail of the CV profiles did not have a great influence on the values of the moments. The extracted projected ranges and standard deviations agree well, as is shown in figs. 4 and 5.

On the other hand the SIMS profiles show "humps". The humps were also observed by Hofker [5] in the tail of the distribution. He concluded that these humps were caused by "preferential penetration of ions which are scattered in the surface layer and are steered into chan- nels in low index directions". This explanation is also applicable here, since the depth of the hump is roughly proportional to the square root of the implantation energy. In the profiles presented in fig. 2 the size of the humps is not the same for every profile. This is probably caused by the fact that the orientation of the wafers during implantation was not the same, due to deviations in the surface orientation and the twist angle. Hofker used samples that were carefully aligned in a dense crystallographic direction.

The humps are most pronounced in the $400 \mathrm{keV}$ (not shown) and $500 \mathrm{keV}$ profiles. In these profiles also humps are observed at the surface side of the distribution. This might be explained by the fact that part of the channeled boron ions is reflected and scattered back in channels as well. The distance travelled by the backscattered boron and the channeled boron is approximately the same, assuming backscattering from the vicinity of the peak of the damage distribution. This peak is located just in front of the peak of the impurity distribution [15].

In figs. 4 and 5 the projected range and the projected range standard deviaton are plotted as a function of implantation energy. In these figures the experimentally obtained data are compared with tabulated data from Smith [16] and Gibbons et al. [17]. These tabulated data are indicated with " $S$ " and " $G$ " respectively. The curve indicated with " $D$ " is the result of a fit with the 


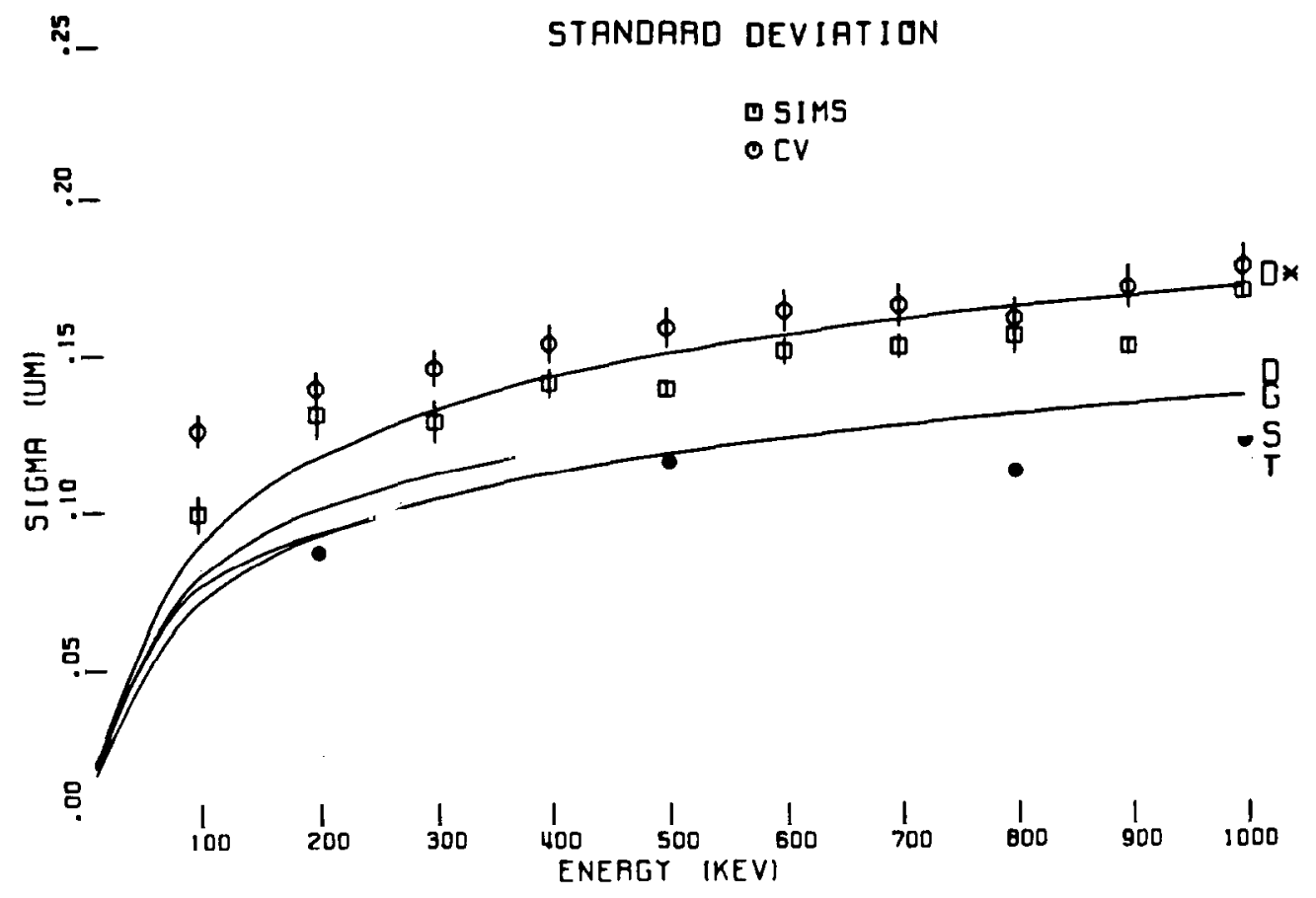

Fig. 5. Projected range standard deviation of boron in silicon as a function of implantation energy, compared with tabulated data $S$ : Smith [16], G: Gibbons et al. [17] D: DIMUS fit $k / k_{\mathrm{L}}=1.57, p=0.49, \mathrm{D}^{*}$ : DIMUS fit $k / k_{\mathrm{L}}=1.57, p=0.465$. T: TRIM 85 calculations.

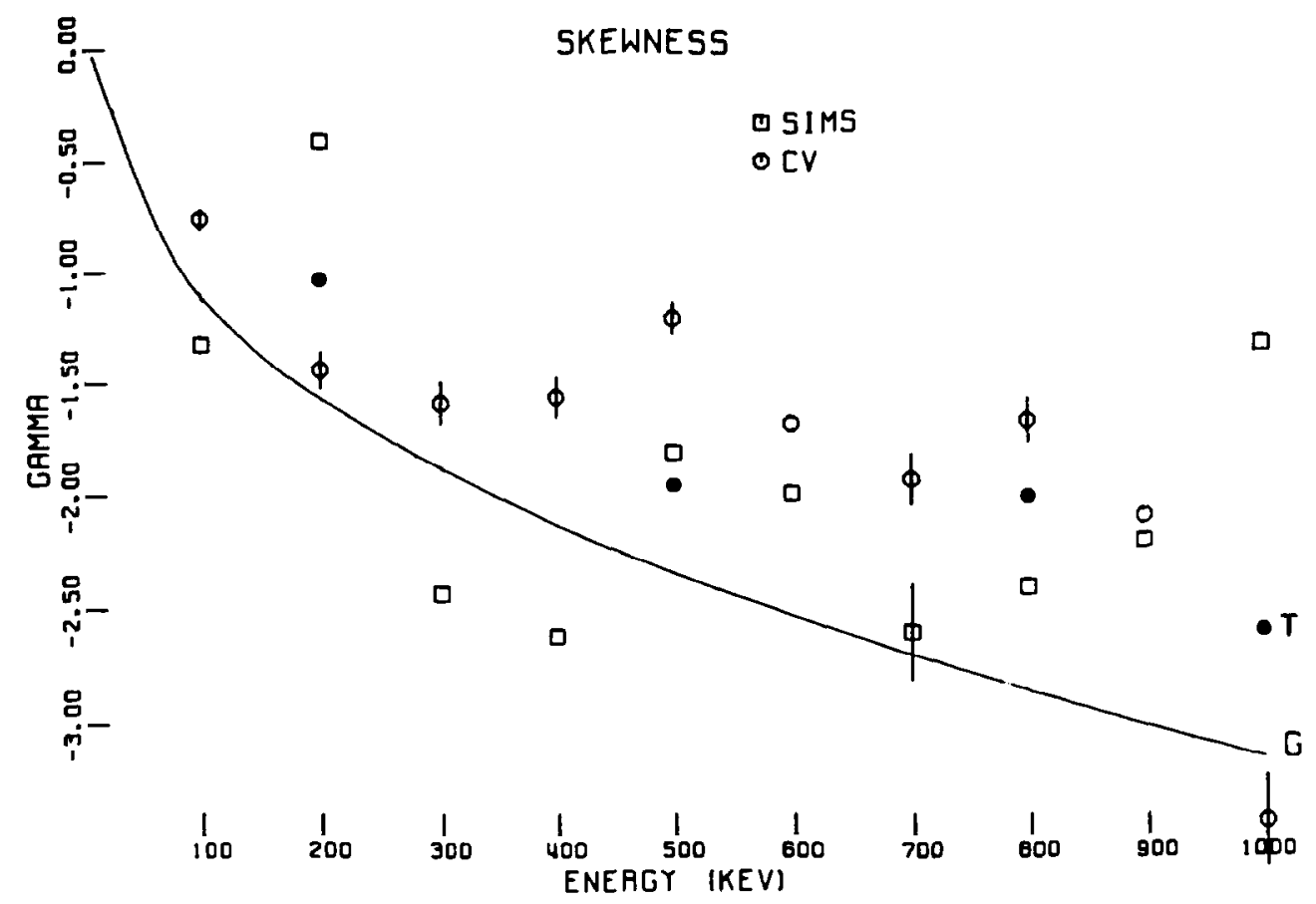

Fig. 6. Skewness of boron as a function of energy compared with estimated values from Gibbons et al. [17]. T: TRIM 85 calculations. 
computer program DIMUS [18] using an electronic stopping of the form:

$S_{\mathrm{e}}=1.57 k_{\mathrm{L}} E^{0.49}$,

where $k_{\mathrm{L}}$ is the Lindhard proportionality constant according to the LSS theory [19], and $E$ the particle energy. The filled circles are data points that result from Monte Carlo simulations using TRIM 85 [19] with a Lindhard correction factor of 1.57 . The experimental values of the projected range agree well with the tabulated data in this energy range, however the TRIM data appear to deviate at higher energies. The $200 \mathrm{keV}$ SIMS data clearly deviate from the expected data. We assume that this is caused by channeling due to a misorientation of the wafer during implantation. This does not hold for the values of the standard deviation in fig. 5 . The calculated data are too low compared with experimental results. Fitting with DIMUS yields an energy dependence of the electronic stopping of the power 0.465 . The agreement of this fit, which is indicated in fig. 5 by $D^{*}$, to the experimental data is rather poor in the low-energy region. These DIMUS fits do not have a direct physical meaning, but are merely presented as a set of data that can easily be obtained and give a fairly good description of the experimental data.

The standard deviation data extracted from CV measurements are systematically somewhat larger than the SIMS data. This means that the profiles determined from $\mathrm{CV}$ are broader than the profiles determined with
SIMS. This broadening is the result of two contributions. The first one, a real broadening, is caused by the fact that the samples for the $\mathrm{CV}$ measurements had to be annealed in order to activate the charge carriers. This annealing step causes an enhanced diffusion due to the implantation damage. The second one is caused by the calculation method. Here part of the broadening is caused by the influence of the Debye length that brings about a greater inaccuracy in the position towards the lower concentrations [21], which leads to broader profiles.

The data for the third moment, the skewness, have smaller values than the tabulated data from Gibbons et al. [17], who give only a "third moment estimate". However, the same tendency is observed. In this case the TRIM data agree well with the expcrimental data. This can be concluded from fig. 6 where the skewness is plotted as a function of implantation energy.

No data will be presented for the fourth moment, the kurtosis, because these data did not show any energy dependence. Calculations show that their influence on the fit is very small, as long as the value of the kurtosis $\beta$ has at least twice the value of $\beta_{\min }$, the minimum value of the kurtosis to get a Pearson type IV distribution [6].

In fig. 7 the mean ranges obtained by the NRA method are compared with the mean ranges deduced from SIMS and CV profiles. From this figure it can be concluded that the data obtained by NRA agree well

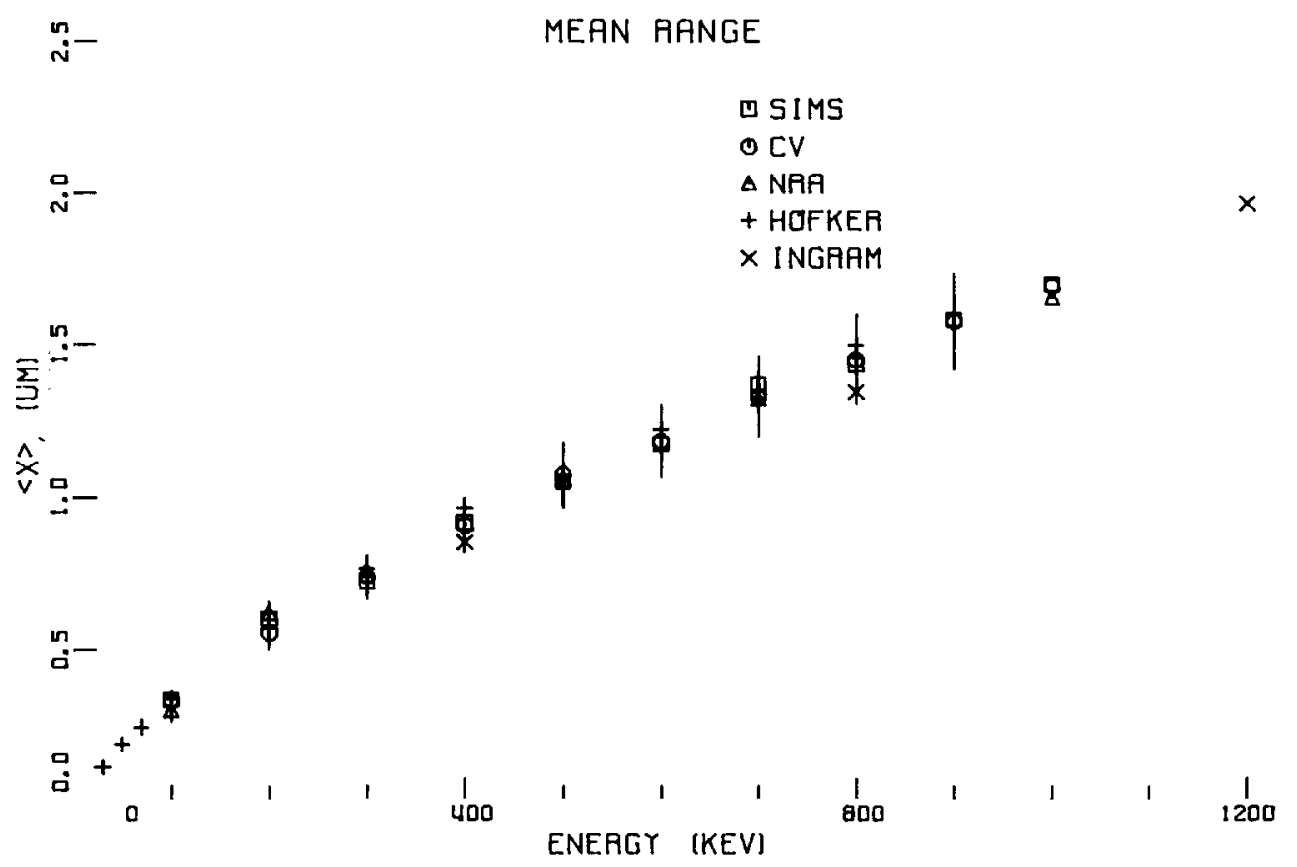

Fig. 7. Comparison of mean ranges of boron in silicon as determined by SIMS, CV and NRA measurements. Also included are data from Hofker [5] and Ingram [22]. 


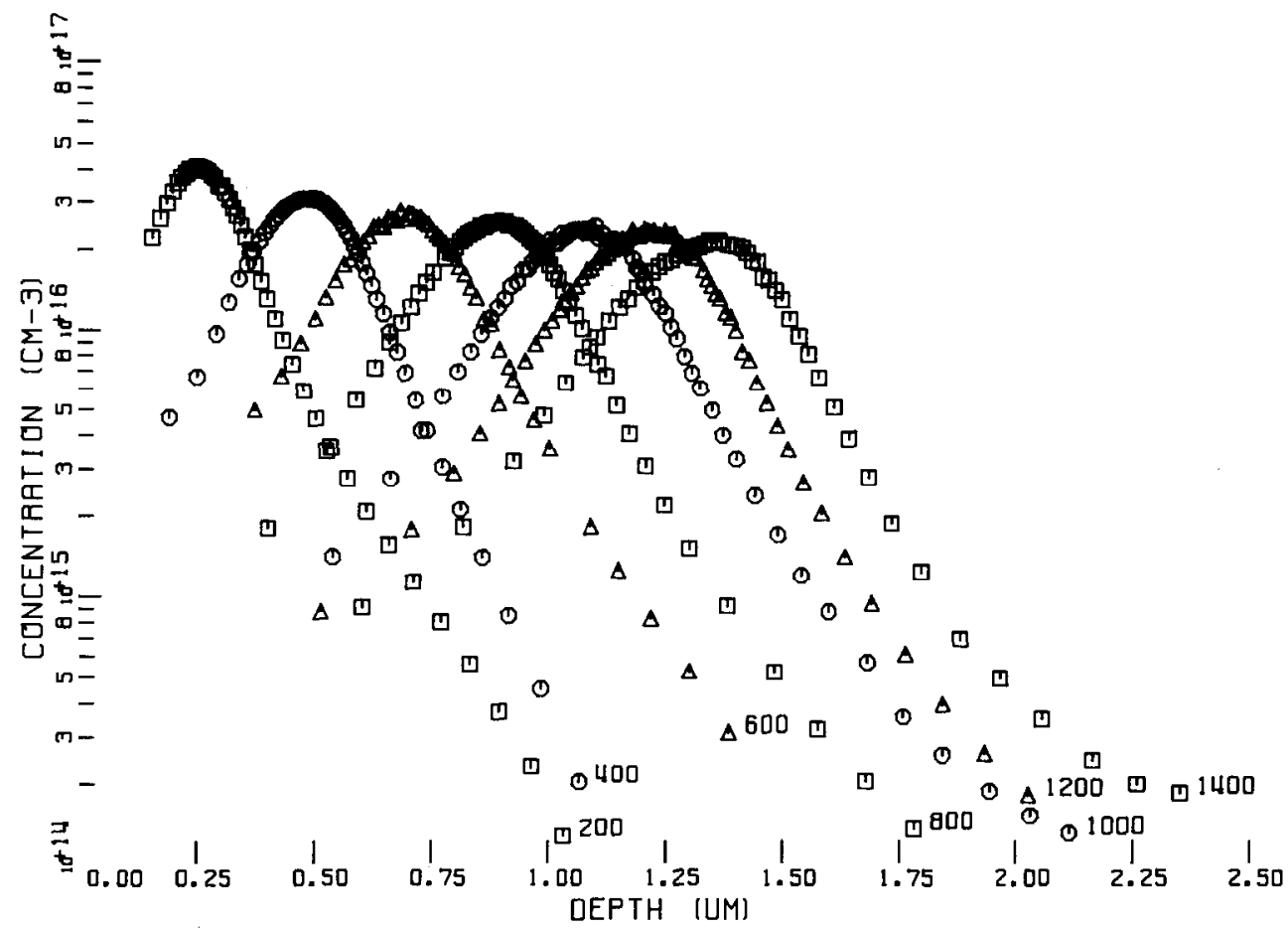

Fig. 8. Impurity profiles of $10^{12} / \mathrm{cm}^{2} 200,400,600,800,1000,1200$ and $1400 \mathrm{keV}$ phosphorus implanted in monocrystalline silicon as determined from $\mathrm{CV}$ measurements.

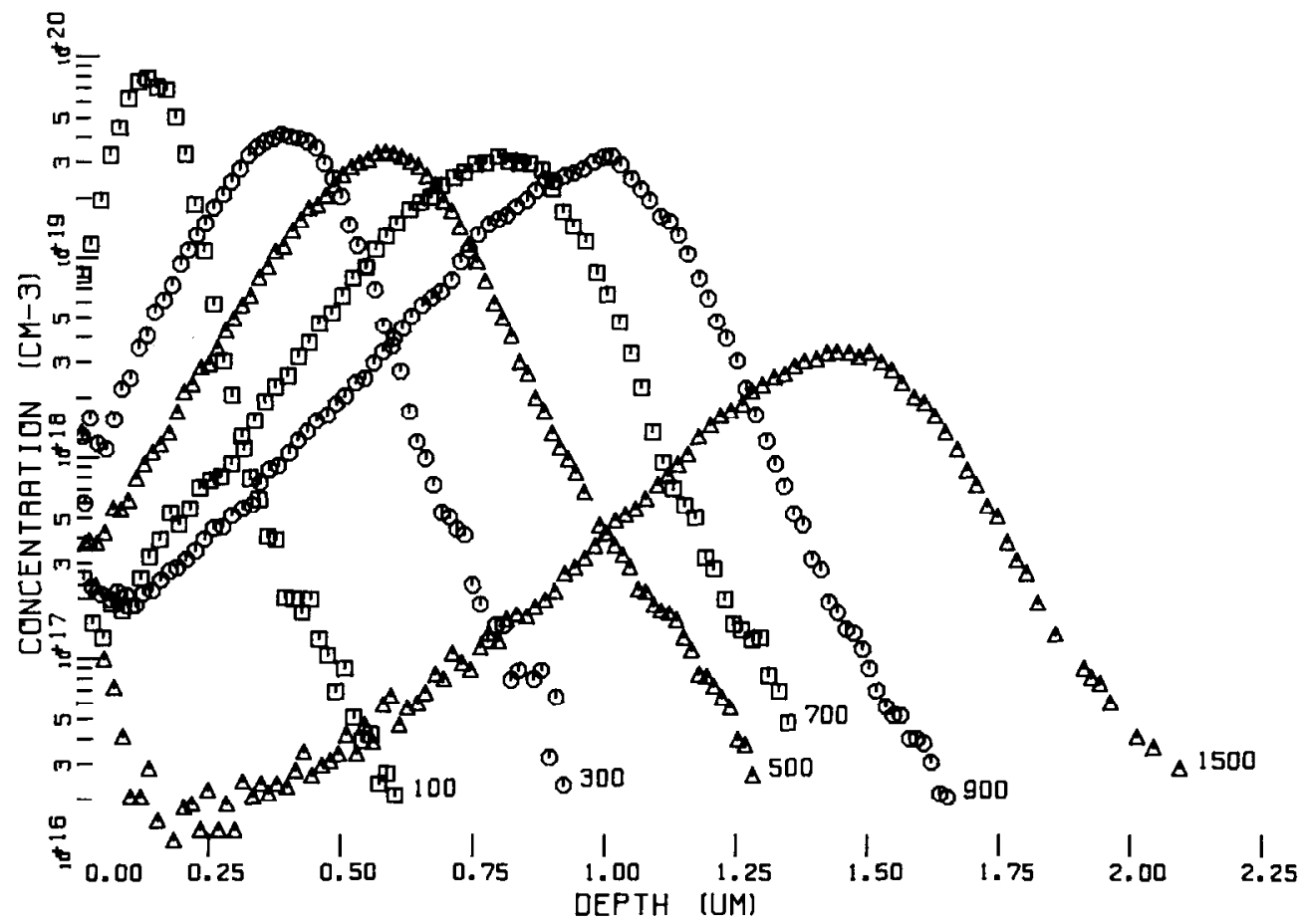

Fig. 9. Impurity profiles of $10^{15} / \mathrm{cm}^{2} 100,300,500,700,900$, and $1.3 \times 10^{14} / \mathrm{cm}^{2} 1500 \mathrm{keV}$ phosphorus implanted in monocrystalline silicon as determined by SIMS. 
with the data obtained by SIMS and CV. Also included in this figure are the data from Hofker [5] for boron implanted in monocrystalline silicon and data recently published by Ingram et al. [22]. As can be seen from this figure all data agree within experimental error although the data from Ingram are slightly lower than the data presented here.

\subsection{Phosphorus implantations}

In figs. 8 and 9 the profiles of phosphorus are presented as obtained from CV and SIMS measurements. Since the ion source allows the formation of $\mathrm{P}^{3+}$, the energy range is extended to $1500 \mathrm{keV}$. The low available current did not cause problems for the doses for $\mathrm{CV}$ measurements in the range up to $1.5 \mathrm{MeV}$. However SIMS measurements require higher doses, we therefore prepared only one high dose sample at 1.5 $\mathrm{MeV}$.

The $100 \mathrm{keV}$ phosphorus CV profile could not be determined. it is so close to the surface that a reliable result could not be obtained using the method as described in sect. 2.2.

The tails in the phosphorus profiles are more pronounced than the tails in the boron distributions. The critical angle for channeling for phosphorus is larger than it is for boron [5]. Therefore boron has a smaller probability of being steered into channels than phos- phorus. The humps observed in the phosphorus SIMS profiles are less pronounced or even absent. This is probably caused by the fact that phosphorus creates more damage than boron and therefore the probability of scattering into low index crystal directions at small depths is low.

In fig. 10 the projected ranges obtained experimentally are compared with tabulated data from Gibbons et al. [17] and Smith [16]. In the energy range considered here, the data agree reasonably well for energies up to $400 \mathrm{keV}$ but deviations are visible for higher energies. The TRIM calculations lead to lower values, while the tables give slightly higher projected ranges, compared to the experimental results. Here again, a Lindhard correction factor of 1.57 is assumed. The experimental data can be fitted using an electronic stopping power model in DIMUS [18] of the form:

$S_{\mathrm{e}}=1.57 k_{\mathrm{L}} E^{0.463}$.

However, it is not possible to fit the projected range standard deviation data with the same model. The ratio $k / k_{\mathrm{L}}$ and the power of $E$ had to be chosen as 0.9 and 0.5 , respectively. This fit is indicated in fig. 11 by $D^{*}$. The data from Smith [16] agree reasonably well in this case, while the data from Gibbons et al. [17] are obviously too low, and the TRIM data are far below the experimental data. No effort was made to try to fit

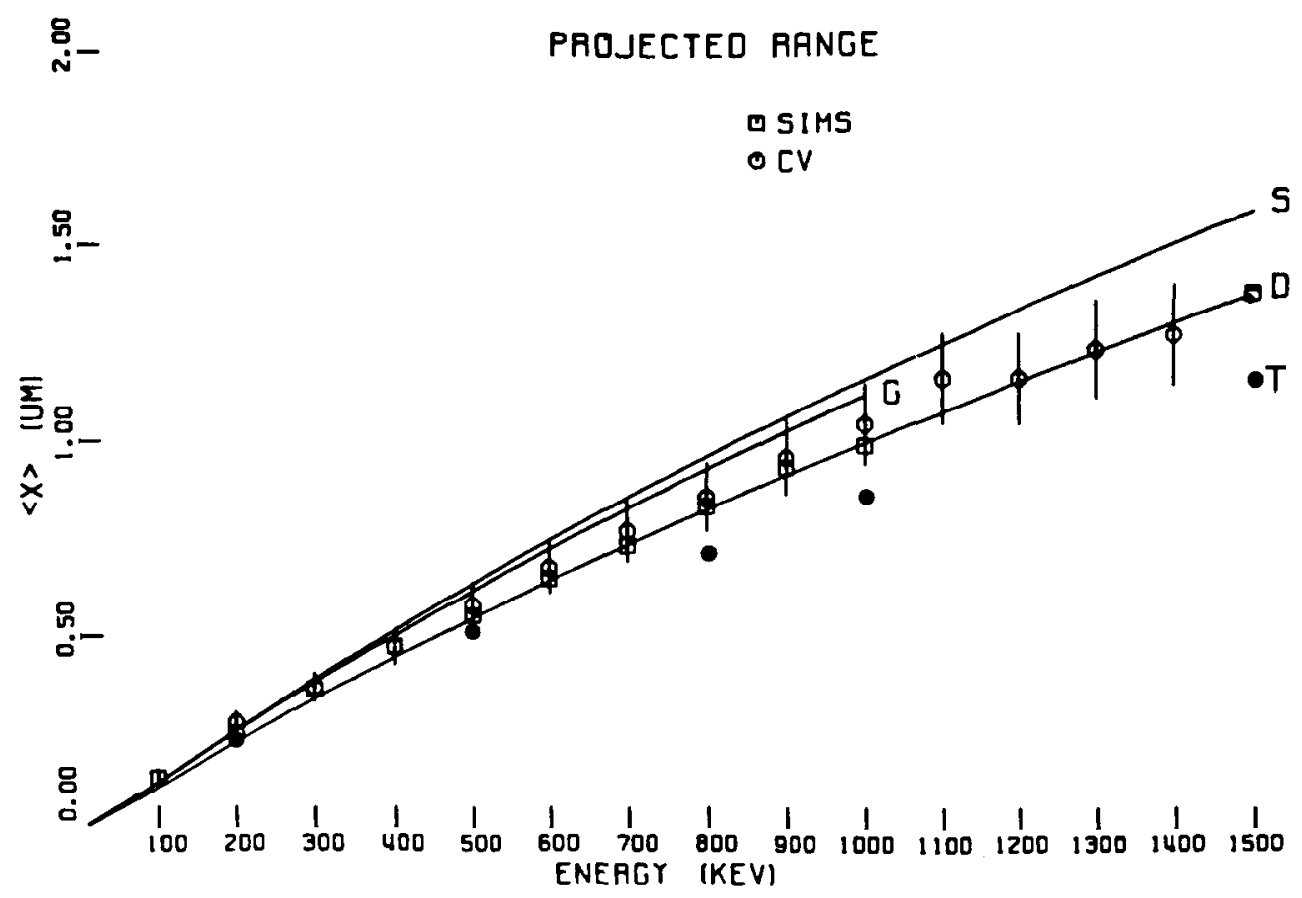

Fig 10. Projected range of phosphorus in silicon as a function of implantation energy, compared with tabulated data: S: Smith [16], G: Gibbons et al. [17], D: DIMUS fit $k / k_{\mathrm{L}}=1.57, p=0.463$, T: TRIM 85 calculations. 


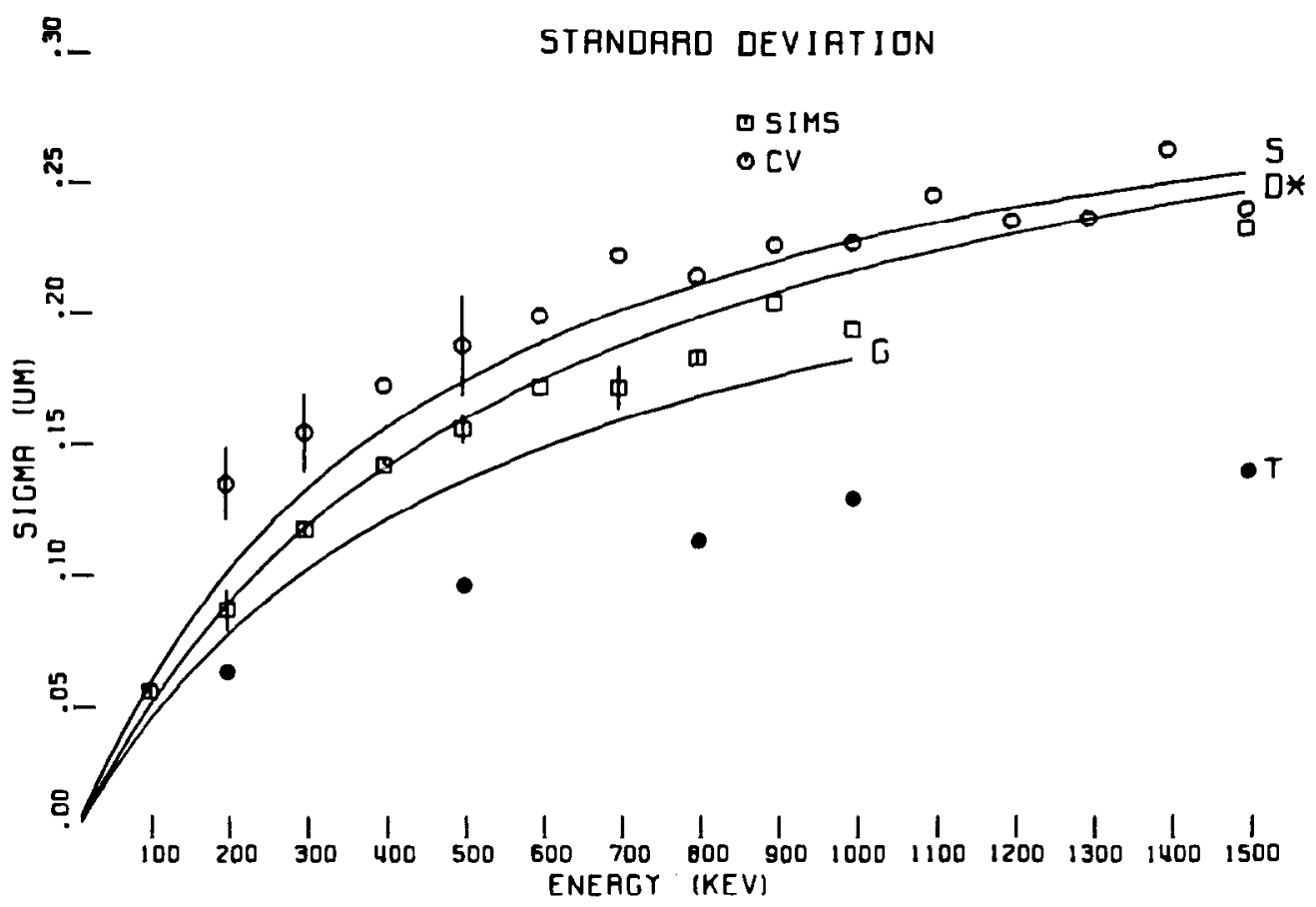

Fig. 11. Projected range standard deviation of phosphorus in silicon as a function of implantation energy, compared with tabulated data: S: Smith [16], G: Gibbons et al. [17], D*: DIMUS fit $k / k_{\mathrm{L}}=0.9, p=0.5$, T: TRIM 85 calculations.

TRIM data to the experimental data by varying input variables. This means that the improvements in the calculation of the standard deviation as proposed by
Smith, seem to be appropriate in the case of phosphorus implantations in silicon.

From this figure, again, it is clear that the $\mathrm{CV}$ data

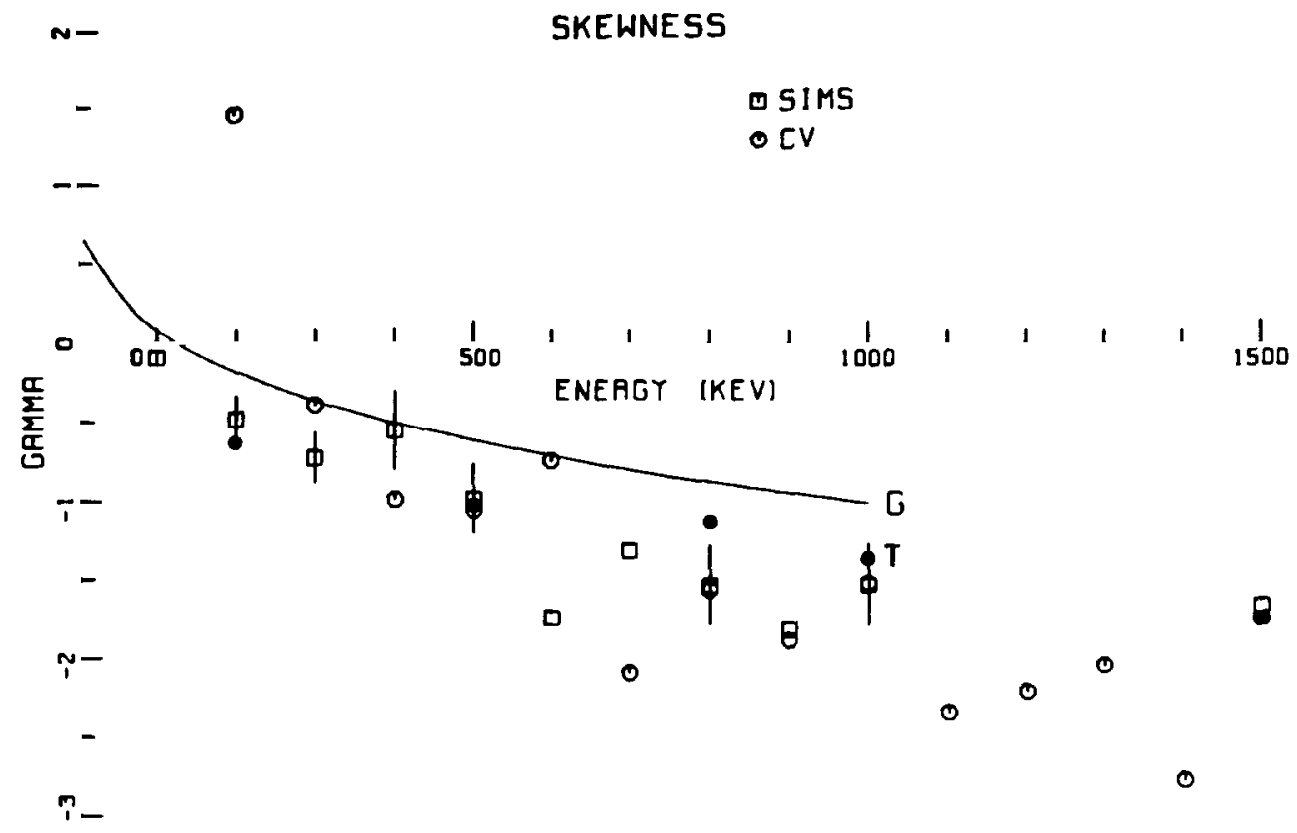

Fig. 12. Skewness of phosphorus as a function of implantation energy, compared with estimated values from Gibbons et al. [17] and TRIM 85 calculations. 


$$
\text { 盇怘一 }
$$

D

ㅁ

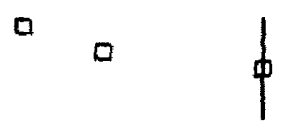

ㅁ-

a

Fig. 13. Kurtosis of phosphorus as a function of implantation energy as determined from SIMS profiles, compared with some TRIM 85 calculations.

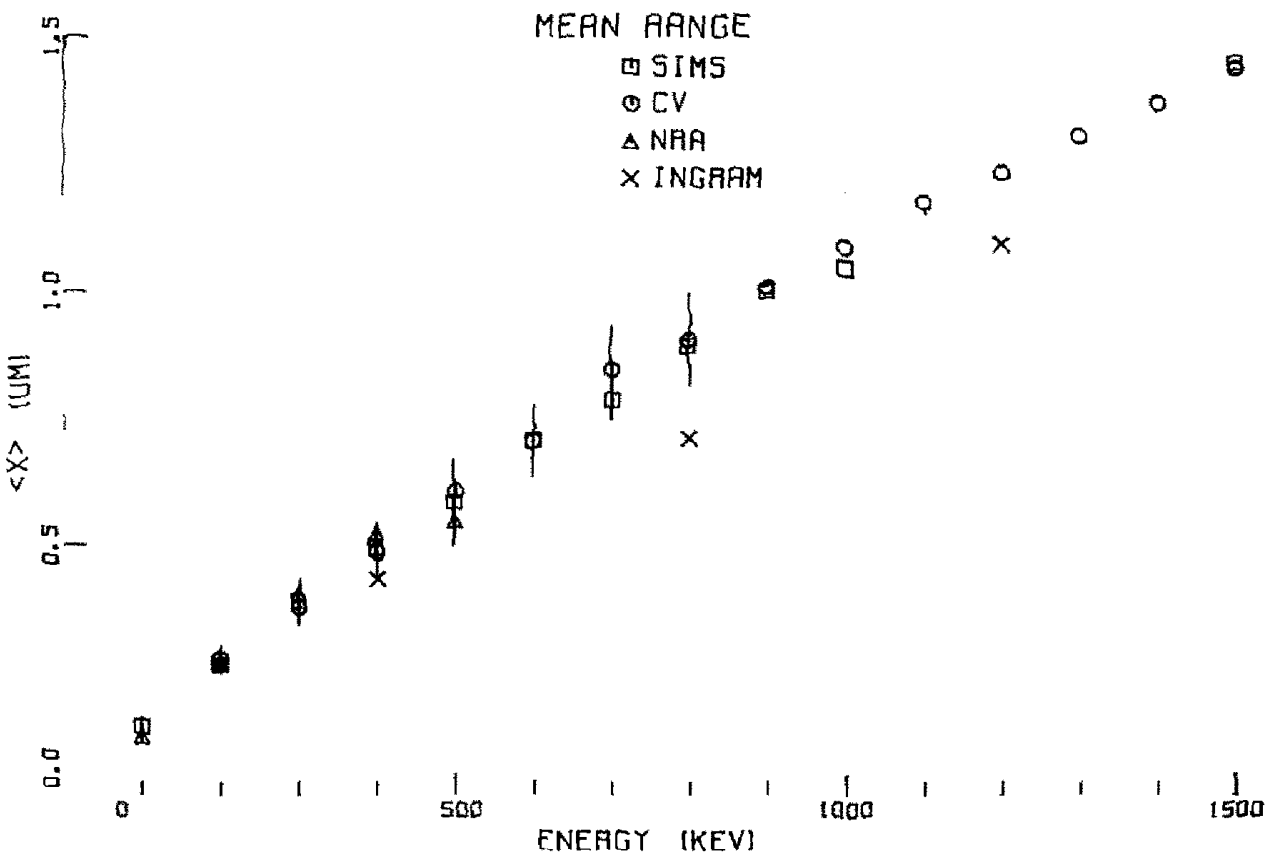

Fig. 14. Comparison of mean ranges of phosphorus in silicon as determined from SIMS, CV and NRA measurements. Also included are data from Ingram [22] 
Table 1

Projected range, projected range straggling and kurtosis for boron and phosphorus as determined from SIMS and CV measurements.

\begin{tabular}{|c|c|c|c|c|c|c|}
\hline \multirow{3}{*}{$\begin{array}{l}E \\
(\mathrm{keV})\end{array}$} & \multicolumn{6}{|l|}{ Boron } \\
\hline & \multicolumn{3}{|l|}{$\mathrm{CV}$} & \multicolumn{3}{|l|}{ SIMS } \\
\hline & $R_{\mathrm{p}}$ & $\Delta R_{\mathrm{p}}$ & $\gamma$ & $R_{\mathrm{p}}$ & $\Delta R_{\mathrm{p}}$ & $\gamma$ \\
\hline 100 & 0.328 & 0.126 & -0.76 & 0.311 & 0.099 & -1.32 \\
\hline 200 & 0.536 & 0.139 & -1.44 & 0.594 & 0.131 & -0.40 \\
\hline 300 & 0.713 & 0.146 & -1.59 & 0.688 & 0.129 & -2.43 \\
\hline 400 & 0.884 & 0.154 & -1.56 & 0.879 & 0.141 & -2.62 \\
\hline 500 & 1.051 & 0.159 & -1.2 & 1.015 & 0.139 & -1.80 \\
\hline 600 & 1.170 & 0.164 & -1.68 & 1.153 & 0.151 & -1.99 \\
\hline 700 & 1.300 & 0.165 & -1.92 & 1.331 & 0.152 & -2.60 \\
\hline 800 & 1.422 & 0.161 & -1.66 & 1.399 & 0.156 & -2.40 \\
\hline 900 & 1.536 & 0.171 & -2.08 & 1.54 & 0.152 & -2.19 \\
\hline 1000 & 1.623 & 0.178 & -3.43 & 1.678 & 0.169 & -1.32 \\
\hline
\end{tabular}

1100

1200

1200

1400

1500

\begin{tabular}{llllll} 
Phosphorus & & & \\
CV & & & \multicolumn{2}{l}{ SIMS } & \\
$R_{\mathrm{p}}$ & \multicolumn{1}{l}{$R_{\mathrm{p}}$} & $\gamma$ & $R_{\mathrm{p}}$ & $\Delta R_{\mathrm{p}}$ & $\gamma$ \\
& & & 0.137 & 0.057 & -0.09 \\
0.276 & 0.135 & 1.47 & 0.248 & 0.087 & -0.48 \\
0.368 & 0.154 & -0.39 & 0.363 & 0.117 & -0.72 \\
0.473 & 0.173 & -0.98 & 0.475 & 0.142 & -0.55 \\
0.582 & 0.188 & -1.05 & 0.556 & 0.156 & -0.98 \\
0.681 & 0.199 & -0.74 & 0.652 & 0.172 & -1.74 \\
0.777 & 0.222 & -2.09 & 0.740 & 0.172 & -1.31 \\
0.863 & 0.214 & -1.56 & 0.838 & 0.183 & -1.53 \\
0.963 & 0.227 & -1.88 & 0.937 & 0.205 & -1.81 \\
1.049 & 0.227 & -1.51 & 0.995 & 0.195 & -1.53 \\
1.169 & 0.246 & -2.34 & & & \\
1.170 & 0.236 & -2.20 & & & \\
1.246 & 0.237 & -2.03 & & & \\
1.284 & 0.263 & -2.76 & & & \\
1.386 & 0.240 & -1.74 & 1.39 & 0.233 & -1.65
\end{tabular}

result in larger standard deviations than the SIMS data.

The SIMS data of the standard deviation match closely with the DIMUS data in the low energy range. This can be explained by the fact that the phosphorus dose used is sufficient to cause an amorphous layer. This means that the LSS theory [19] is valid and Gaussian distributions result. The argument is strengthened by the data presented in figs. 12 and 13 where the skewness and the kurtosis are plotted as a function of implantation energy. In the lower energy region the skewness is close to zero and the kurtosis close to 3 . When these values for the third and fourth moment are used in a Pearson IV distribution, a Gaussian distribution results [6]. The TRIM results of the skewness match closely with the experimental data, as can be seen in fig. 12. The TRIM data of the kurtosis show the same trend as the experimental data.

The data for the kurtosis obtained from CV measurements are not shown herc becausc they did not show any energy dependence.

In fig. 14 the mean range as obtained from NRA measurements is compared with $\mathrm{CV}$ and SIMS data. These data agree well. Recently published data from Ingram et al. [22] are included for comparison. These data are lower than our data, which might be attributed of the profiling method. Ingram used the spreading-resistance method with multi-layer correction.

In table 1 the results as presented in figs. $4-6,10,11$ and 12 are summarized.

\section{Conclusions}

Three different techniques have been used to determine the implantation profiles of boron and phos- phorus in silicon: SIMS, CV and NRA. As can be concluded from mean range measurements the results from these three techniques agree well within experimental error.

The profiles obtained from $\mathrm{CV}$ measurements show penetrating tails that are (at least partly) caused by the used measuring technique. The corresponding SIMS profiles show tails that are less pronounced or even absent.

The phosphorus profiles show more pronounced tails than the boron distributions. This is caused by channeling resulting from scattering into low index directions almost at the end of the trajectory.

The boron SIMS profiles show humps that are formed by channeled boron ions that have been steered into low index directions close to the surface. This can be concluded from the fact that the depth of the humps is roughly proportional to the square root of the implantation energy. Part of the channeled boron is backscattered in the vicinity of the peak in the damage distribution. This can be concluded from the location of a second hump on the surface side of the distribution.

These humps are less pronounced or even absent in the phosphorus profiles obtained by SIMS. This is explained by the fact that the phosphorus dose caused enough damage to dechannel a large fraction of the channeled phosphorus.

The implantation profiles in the energy range investigated, can be described by four moments using a Pearson IV distribution, of which the fourth moment is dependent on the value of the third moment.

For boron the projected ranges agree well with tabulated data, but can be best described using an electronic stopping power proportional to (energy) ${ }^{0.49}$. 
For phosphorus the tabulated data for the projected range are too high for energies beyond $400 \mathrm{keV}$ while TRIM 85 calculations show results that are too low for energies beyond $400 \mathrm{keV}$. The experimental data can be best described using an electronic stopping power proportional to (energy) ${ }^{0.463}$.

The CV profiles are markedly broader than the SIMS profiles as can be concluded from the presented standard deviation data. This broadening is caused partly by enhanced diffusion that occurs when the samples are annealed to activate the charge carricrs and partly by the calculation method.

Theoretical standard deviation data are too low for boron, but in the case of phophorus corrections made by Smith appear to lead to agreement between tabulated and experimental data.

Tabulated data for the third moment ratio appear to show the same trend as the experimental data but are too small for phosphorus and too large for boron compared to these data.

The TRIM 85 calculations show deviations from the experimentally determined data for the projected range, especially in the high energy range, while for the projected range straggling TRIM 85 data are clearly too low. However, the TRIM calculations for the third and fourth moment show reasonable agreement with the experimental data.

These investigations in the program of the Foundation for Fundamental Research on Matter (FOM) have been supported by the Foundation for Technical Research (STW), Technical Science Branch/Division of the Netherlands Organization for the Advancement of Pure Research (ZWO).

Furthermore the author gratefully acknowledges the assistance of $\mathrm{K}$. Lippc with CV measurements, the assistance of M. Vos, D.O. Boerma and P.J.M. Smulders with the NRA measurements, and the help of P. von Rosenstiel for intermediating in the contacts with Western University. A. Stolmeijer and Ph. Wolbert are gratefully acknowledged for writing the fit program IMPFIT, and J. Middelhoek is gratefully acknowledged for discussing the manuscript.

\section{References}

[1] D. Pramanik and M.I. Current, Solid-State Techn. 27 (1984) 211.
[2] K.W. Terrill, P.F. Byrnc, C. Hu and N.W. Cheung, Appl. Phys. Lett. 45 (1984) 977.

[3] M.I. Current, R.A. Martin, K. Doganis and R.H. Bruce, Semicond. Int., 8 (6) (1985) 106.

[4] A. Stolmeijer, Ph.D. Thesis Twente University of Technology, Enschede, The Netherlands (1986).

[5] W.K. Hofker, Philips Res. Rep. Suppl. No. 8 (1975).

[6] H. Ryssel, G. Prinke, K. Hoffmann, K. Haberger, K. Mueller and R. Henkelmann, Appl. Phys. 24 (1981) 39.

[7] D.A. Antoniades, S.E. Hansen and R.W. Dutton, SUPREM II, Technical Report No. 5019-2, Sel 78-020, Stanford Electronics Labs (1978).

[8] H. Ryssel, K. Haberger, K. Hoffmann, G. Prinke, R. Dumcke and A. Sachs, IEEE Trans. Electron Devices ED-27 (1980) 1484.

[9] N.L. Turner, M.I. Current, T.C. Smith and D. Crane, Solid-State Techn. 28 (1985) 163.

[10] S. Oosterhoff and J. Middelhoek, Solid-State Electron. 28 (1985) 427.

[11] M. Vos, D.O. Boerma, P.J.M. Smulders and S. Oosterhoff, Nucl. Instr. and Meth. B17 (1986) 234.

[12] R. Fletcher, A modified Marquardt Subroutine for nonlinear least squares, Harwell Report AERA R 6799, Harwell (1971).

[13] T.E. Seidel, in: 2nd Int. Conf. on Ion Implantation in Semiconductors, eds. I. Ruge and J. Graul (Springer, Berlin, 1971) p. 47.

[14] C.P. Wu, R.C. Douglas and C.W. Muller, IEEE Trans. Electron Devices ED-22 (1975) 319.

[15] S. Oosterhoff, Ph.D. Thesis Twente University of Technology, Enschede, The Netherlands (1986).

[16] B.J. Smith, Ion Implantation Range Data for Silicon and Germanium Device Technologies (Learned Information, Oxford, 1977).

[17] J.F. Gibbons, W.S. Johnson and S.W. Mylroie, Projected Range Statistics (Academic Press, Stroudsburg, 1975).

[18] J.P. Biersack and J.F. Ziegler, in: Ion Implantation Techniques, Springer Series in Electrophysics, vol. 10, eds. $\mathbf{H}$. Ryssel and H. Glawischnig (Springer, Berlin, 1982) p.157.

[19] J. Lindhard, M. Scharff and H.E. Schiøtt, K. Dan. Vidensk. Selsk. Matt. Fys. Medd. 33 (1963) no. 1.

[20] J.F. Ziegler, J.P. Biersack and U. Littmark, The stopping and range of ions in solids, in: The stopping and range of ions in matter, vol. 1, ed. J.F. Ziegler (Pergamon, New York, 1985).

[21] S.M. Sze, Physics of Semiconductor Devices, 2nd ed. (Wiley, New York, 1981).

[22] D.C. Ingram, J.A. Baker and D.A. Walsh, Nucl. Instr. and Meth. B7/8 (1985) 361. 